\title{
Molecular Detection of Anaplasma spp. and Ehrlichia spp. in Ruminants from Twelve Provinces of China
}

\author{
Haixiang Qiu, ${ }^{1}$ Patrick John Kelly, ${ }^{2}$ Jilei Zhang, ${ }^{1}$ Qinghua Luo, ${ }^{1}$ Yi Yang,

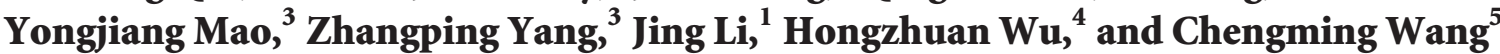 \\ ${ }^{1}$ Jiangsu Co-Innovation Center for the Prevention and Control of Important Animal Infectious Diseases and Zoonoses, \\ Yangzhou University College of Veterinary Medicine, Yangzhou, Jiangsu 225009, China \\ ${ }^{2}$ Ross University School of Veterinary Medicine, Basseterre 00265, Saint Kitts and Nevis \\ ${ }^{3}$ Yangzhou University College of Animal Science and Technology, Yangzhou, Jiangsu 225009, China \\ ${ }^{4}$ Alabama State University, Montgomery, AL 36104, USA \\ ${ }^{5}$ Department of Pathobiology, Auburn University College of Veterinary Medicine, Auburn, AL 36849, USA
}

Correspondence should be addressed to Chengming Wang; wangche@auburn.edu

Received 31 October 2016; Accepted 27 November 2016

Academic Editor: Matilde J. Coello

Copyright (C) 2016 Haixiang Qiu et al. This is an open access article distributed under the Creative Commons Attribution License, which permits unrestricted use, distribution, and reproduction in any medium, provided the original work is properly cited.

\begin{abstract}
Anaplasma spp. and Ehrlichia spp. are tick-transmitted bacteria that are of significant economic importance as they can infect large and small ruminants and also people. There is little information on anaplasmosis and ehrlichiosis in ruminants in China. 16S rRNA FRET-qPCRs were used to screen convenience whole blood samples from 2,240 domestic ruminants in 12 provinces of China for Anaplasma spp. and Ehrlichia spp. Positive samples were further analyzed with a standard PCR for the gltA. Anaplasma spp. DNA was detected in the sheep $(11.7 \% ; 13 / 111)$, goats $(81.8 \% ; 219 / 270)$, cattle $(13.2 \% ; 241 / 1,830)$, and water buffaloes $(6.9 \% ; 2 / 29)$. Ehrlichia spp. DNA was detected in sheep $(1.8 \% ; 2 / 111)$, goats $(1.1 \% ; 3 / 270)$, and cattle $(3.6 \% ; 65 / 1830)$ but not in water buffaloes (0/29). Sequencing of gltA PCR products showed that A. marginale, A. ovis, Ehrlichia canis, and Ehrlichia sp. (JX629807) were present in ruminants from China, while the 16S rRNA FRET-qPCR sequence data indicated that there might also be $A$. platys, A. phagocytophilum, Anaplasma sp. BL126-13 (KJ410243), and Anaplasma sp. JC3-6 (KM227012). Our study shows that domestic ruminants from China are not uncommonly infected with a variety of Anaplasma spp. and Ehrlichia spp.
\end{abstract}

\section{Background}

Anaplasma spp. and Ehrlichia spp. are tick-transmitted, intracellular Gram-negative bacteria that are important animal and human pathogens. The major Anaplasma species that impact animal and human health are Anaplasma marginale, A. ovis, A. centrale, A. bovis, A. phagocytophilum, and A. platys [1]. The most important of these in ruminants is A. marginale which causes bovine anaplasmosis (formerly gall-sickness) which is associated with fever, anemia, icterus, and often death. The major pathogenic Ehrlichia species are E. canis, E. chaffeensis, E. ewingii, E. muris, and E. ruminantium [2] with the latter causing heartwater in domestic ruminants. This disease is prevalent in Africa, where it causes high mortality (up to 90\%) and extensive economic losses [3].
In China, there is little information on the Anaplasma and Ehrlichia species in domestic ruminants. Anaplasma bovis, A. marginale, and A. ovis have been described in various Provinces, including Xinjiang, Gansu, Henan, Yunnan, Hubei, Guizhou, and Zhejiang [1,4-7]. In addition, $A$. phagocytophilum has been reported in ruminants (sheep and cattle) in Henan and Xinjiang as well as in dogs and ticks $[1,6-14]$, and A. platys has been detected in red deer and sika deer from Gansu province [4]. In the case of Ehrlichia spp. in ruminants in China, an unclassified species has been reported in cattle in Tibet [15] and E. canis, a species that infects dogs worldwide, has been reported in sika deer from Gansu [4].

To provide further information on Anaplasma spp. and Ehrlichia spp. infections in domestic ruminants of China, we carried out a PCR survey for the organisms in blood samples 
from ruminants in 12 provinces of China. Our findings are reported below.

\section{Materials and Methods}

2.1. Blood Samples. This study was reviewed and approved by the Institutional Animal Care and Use Committee of Yangzhou University and animal owners gave written permissions for blood collection.

Between 2007 and 2013, convenience whole blood samples (around $6 \mathrm{~mL}$ ) from cattle $(n=1,830)$, water buffaloes $(n=29)$, goats $(n=270)$, and sheep $(n=111)$ were collected in 12 provinces/municipalities of China as described before $[16,17]$ (Table 1). DNA was extracted from the whole blood samples using a standard phenol-chloroform method as described previously [16] and stored at $-80^{\circ} \mathrm{C}$ until PCRs were performed.

2.2. FRET-qPCR. As described previously, FRET-qPCRs for the 16S rRNA of Anaplasma spp. [18] and Ehrlichia spp. [19], and the mammalian HMBS gene [20] as an endogenous internal control, were performed on a Roche Light-Cycler 480-II PCR Instrument. Positive PCR products were verified by gel electrophoresis and sequenced using forward and antisense primers (BGI, Shanghai, China). Negative controls consisting of sterile molecular grade water were used to detect cross-contamination during DNA extraction and PCR processing.

2.3. Standard PCR for the Citrate Synthase Gene (gltA). To further characterize the Anaplasma and Ehrlichia species detected above, we carried out standard PCRs for the citrate synthase gene ( $g l t A)$ of Anaplasma spp. as described previously [21] and for Ehrlichia spp. with primers designed for the study (forward primer: GGTTTATGGTGCTTTTCCTAGTGTTGA; reverse primer: TTACAGATTTCTCAGGAGTATATGCCTCC). The PCR products we obtained were verified by gel electrophoresis and sequenced (BGI, Shanghai, China).

2.4. DNA Sequence Data Analysis. Compilation and assembly of the multiple sequences generated from each template were performed using the Vector NTI. Sequence alignment was performed with Align (Vector NTI).

\section{Results}

The mammalian HMBS gene endogenous internal control was positive for all samples, indicating that DNA extraction had been successful.

3.1. Anaplasma spp. DNA in Ruminants. Overall, $17.2 \%$ (385/ 2240) of the ruminants from 8 of the 12 provinces studied (Table 1 and Figure 1) were positive for the 16S rRNA of Anaplasma spp. with copy numbers ranging from 50 to $52,000 / \mathrm{mL}$ blood (median 1,720 copies/mL blood). Goats were most frequently positive $(81.1 \%$; $219 / 270)$, followed by cattle $(13.2 \% ; 241 / 1,830)$, sheep $(11.7 \% ; 13 / 111)$, and water buffaloes $(6.9 \% ; 2 / 29)$.

When we sequenced the positive 16S rRNA FRET-qPCRs, we obtained clean sequencing data for 38 of the samples from cattle (23) and goats (15) (Table 2). The sequences in the cattle were most commonly identical to those of $A$. phagocytophilum (12/23; 52\%) and A. marginale (10/23; 44\%) with positive animals in 4 and 2 of the 9 provinces studied, respectively. Representative sequences were deposited in GenBank for A. phagocytophilum (KX279691) and A. marginale (KX279690). The one other Anaplasma sp. found was in a Wannan cow from Anhui which had a 16S RNA sequence identical to that of a poorly characterized Anaplasma sp. BL126-13 (KJ410243). We also found evidence for this organism in three goats from Jiangsu. Other Anaplasma spp. we detected in goats were A. ovis (KX279688) in Xinjiang (2), A. platys (KX279689) in Jiangsu (2), and a poorly characterized Anaplasma sp. (KM227012) in Jiangsu (5). The 16S rRNA sequences we deposited in GenBank (KX279683; KX279685) are identical to those of Anaplasma sp. (KM227012) and Anaplasma BL126-13 (KJ410243), respectively.

Since there is limited polymorphism in the 16S rRNA FRET-qPCR sequences of different ruminant Anaplasma spp. (Figure 2), to enable more definitive species differentiation we carried out a PCR and sequencing of the more polymorphic $g l t A$ on $20 \%$ of the $16 \mathrm{~S}$ rRNA positive samples with the highest copy numbers (range of 610 to $52,000 / \mathrm{mL}$ blood; median $2,300)$. Only two samples provided clean sequencing data with one (16S rRNA FRET-qPCR copy number 52,000/mL blood) from a bovine in Yunnan having 100\% identity with A. marginale (0/620 mismatches with CP006847.1) and the other (16S rRNA FRET-qPCR copy number 47,700 copies $/ \mathrm{mL}$ blood) from a goat in Xinjiang having $99.7 \%$ identity with $A$. ovis (1/438 mismatches with KJ410284.1). The gltA sequence of $A$. marginale we identified was deposited in GenBank under the accession number KX506005 and that of A. ovis as KX506006. The 16S rRNA sequences for Anaplasma spp. from ruminants in this study are compared with those of other representing Anaplasma spp. (Figure 3).

3.2. Ehrlichia spp. DNA in Ruminants. A total of 70 animals (70/2,240, 3.1\%) were positive for DNA of Ehrlichia spp. in our $16 \mathrm{~S}$ rRNA FRET-qPCR with copy numbers varying from 50 to $42,900 / \mathrm{mL}$ blood (median 5,100 ). Cattle were most frequently positive $(3.6 \%, 65 / 1,830)$ followed by goats $(1.1 \%, 3 / 270)$ and sheep $(1.8 \%, 2 / 111)$. None of the water buffaloes (29) were positive. We found Ehrlichia sp. positive animals in over half (7/12) the provinces we studied with the highest prevalence in Wuhu $(82.4 \%, 14 / 17)$ of Anhui province and lower prevalence in the tropical provinces in the south of China, mainly Hainan $(20.3 \%, 15 / 74)$, Yunnan $(17.9 \%, 30 / 168)$, and Fujian $(8.3 \%$, $2 / 24$ ). No positive animals were detected from the more northern provinces of Beijing, Shanghai, Heilongjiang, and Tianjin (Table 1; Figure 1).

Standard PCR and sequencing of the glt $A$ were performed on samples positive in the FRET-qPCR for 16S rRNA with higher copy numbers, namely, those between 14,400 and $42,900 / \mathrm{mL}$ blood (median 16,700). Useable gltA sequences 


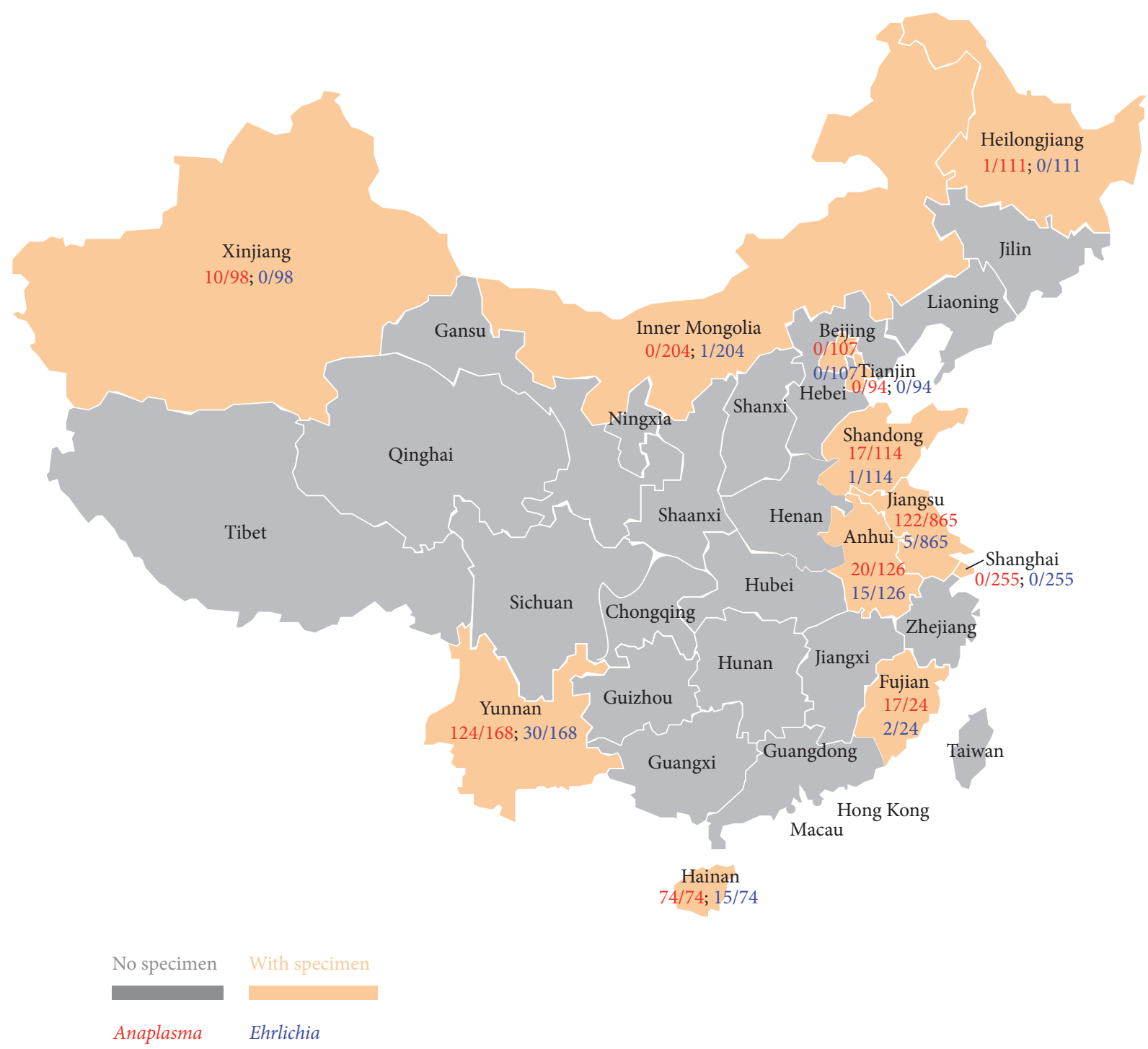

Figure 1: Detection of Anaplasma spp. and Ehrlichia spp. in ruminants from 12 provinces of China. Blood samples of ruminants (2,240) were collected from twelve provinces (in bisque) of China. The prevalence is shown for Anaplasma spp. (red) and Ehrlichia spp. (blue).

were obtained for six of the animals studied (copy numbers 33,100 to $42,900 / \mathrm{mL}$ blood, mean 37,600 ) showing five, all cattle from Yunnan, to be infected with an Ehrlichia sp. having an identical sequence $(563 / 563 ; 100 \%)$ to that of a new species closely related to E. canis and found in Rhipicephalus microplus in the Czech republic (JX629807) [22]. The remaining animal, a goat from Jiangsu, was found to be infected with E. canis having a sequence almost identical $(549 / 563 ; 98 \%)$ to that of E. canis (Oklahoma strain; AF304143) [23] and a strain found in a dog in Thailand (KJ459920) [24].

The gltA sequence of $E$. canis we identified was deposited in GenBank under the accession number KX506008 and that of a representative of the Ehrlichia sp. (JX629807) as KX506006. The gltA sequences for Ehrlichia spp. from ruminants in this study are compared with those of other representing Ehrlichia spp. (Figure 4).

\section{Discussion}

Our results are consistent with other PCR studies from China $[1,6-10,12,13]$ showing that domestic ruminants from the country are infected with a range of Anaplasma and Ehrlichia spp. Differences in the prevalence of animals on the farms we studied were most likely due to differing husbandry practices and tick exposure, with dairy animals and water buffaloes raised intensively having the lowest levels of positivity. Also, although our sample numbers were small, our results indicate that ruminants are generally more commonly infected with Anaplasma and Ehrlichia spp. in the more southern provinces (Yunnan and Hainan) and along the seaboard (Fujian, Anhui, and Jiangsu) where there are more tropical conditions and tick vectors are expected to be more prevalent. Lower prevalence was found in the cooler 
TABle 1: Molecular detection of Anaplasma spp. and Ehrlichia spp. in Chinese ruminants.

\begin{tabular}{|c|c|c|c|c|c|c|}
\hline Animal species & \multicolumn{2}{|c|}{ Subspecies/breed } & Province & City & $\begin{array}{c}\text { Anaplasma positivity } \\
\text { Positive/total, } \%\end{array}$ & $\begin{array}{c}\text { Ehrlichia positivity } \\
\text { Positive/total, \% }\end{array}$ \\
\hline \multirow{11}{*}{ Cattle $(n=1,830)$} & \multirow{11}{*}{ Bos p.taurus } & Holsteins & Anhui & Bengbu & $3 / 109,2.8 \%$ & $1 / 109,0.9 \%$ \\
\hline & & Wannan & Anhui & Wuhu & $17 / 17,100.0 \%$ & $14 / 17,82.4 \%$ \\
\hline & & Holsteins & Beijing & Sanyuan & $0 / 107,0.0 \%$ & $0 / 107,0.0 \%$ \\
\hline & & Holsteins & Jiangsu & Yancheng & $1 / 395,0.3 \%$ & $2 / 395,0.5 \%$ \\
\hline & & Holsteins & Jiangsu & Yangzhou & $0 / 269,0.0 \%$ & $1 / 269,0.4 \%$ \\
\hline & & Holsteins & Heilongjiang & Qiqihar & $1 / 111,0.9 \%$ & $0 / 111,0.0 \%$ \\
\hline & & Simmentals & Inner Mongolia & Chifeng & $0 / 132,0.0 \%$ & $0 / 132,0.0 \%$ \\
\hline & & Luxi & Shandong & Jining & $3 / 42,7.1 \%$ & $0 / 42,0.0 \%$ \\
\hline & & Bohaiblack & Shandong & Binzhou & $1 / 33,3.0 \%$ & $0 / 33,0.0 \%$ \\
\hline & & Holsteins & Shanghai & Shanghai & $0 / 255,0.0 \%$ & $0 / 255,0.0 \%$ \\
\hline & & Holsteins & Tianjin & Tianjin & $0 / 94,0.0 \%$ & $0 / 94,0.0 \%$ \\
\hline \multirow{3}{*}{ Cattle $(n=1,830)$} & \multirow{3}{*}{ Bos p. indicus } & Minnan & Fujian & Putian & $17 / 24,70.8 \%$ & $2 / 24,8.3 \%$ \\
\hline & & Leiqiong & Hainan & Haikou & $74 / 74,100.0 \%$ & $15 / 74,20.3 \%$ \\
\hline & & Yunling & Yunnan & Kunming & $124 / 168,73.8 \%$ & $30 / 168,17.9 \%$ \\
\hline Water buffalo $(n=29)$ & \multicolumn{2}{|c|}{ Haizi } & Jiangsu & Yancheng & $2 / 29,6.9 \%$ & $0 / 29,0.0 \%$ \\
\hline \multirow{2}{*}{ Goats $(n=270)$} & \multirow{2}{*}{\multicolumn{2}{|c|}{$\begin{array}{c}\text { Yangtze River Delta White } \\
\text { Xinjiang }\end{array}$}} & Jiangsu & Yangzhou & $119 / 172,69.2 \%$ & $3 / 172,1.7 \%$ \\
\hline & & & Xinjiang & Urumqi & $10 / 98,10.2 \%$ & $0 / 98,0.0 \%$ \\
\hline \multirow{2}{*}{ Sheep $(n=111)$} & \multicolumn{2}{|c|}{ Sishui Fur } & Inner Mongolia & Xilingol & $0 / 72,0.0 \%$ & $1 / 72,1.4 \%$ \\
\hline & \multicolumn{2}{|c|}{ Wuranke } & Shandong & Jining & $13 / 39,33.3 \%$ & $1 / 39,2.6 \%$ \\
\hline
\end{tabular}

\begin{tabular}{|c|c|}
\hline A. bovis KP314249 & AAAACCTTACCACCCCTTGACATGAAGATTAGTTCCTCCTTAACAGGAGGGCGCAGTTAGGCTGGGTCTTGCA \\
\hline A. marginale СР006847 & 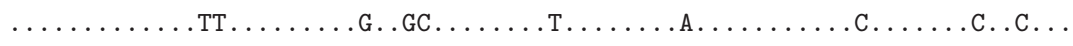 \\
\hline A. marginale KX279690 & 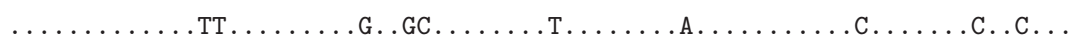 \\
\hline A. phagocytophilum KJ782389 & .С........... \\
\hline A. phagocytophilum KX279691 & . .TT............... \\
\hline A. platys KJ459912 & ..G.......TT......................... \\
\hline A. platys KX279689 & .G........TT.......................... \\
\hline A. ovis $\mathrm{KJ} 410245$ & .......... TT.......G.GC................ \\
\hline A. ovis KX279688 & ...TT.......G.GC............G.A... \\
\hline A. sp. KJ410243 & ..T. \\
\hline A. sp. KX279685 & .T. . \\
\hline A. sp. KM227012 & 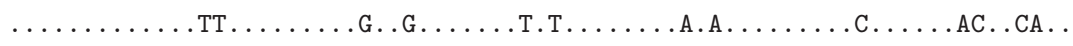 \\
\hline A. sp. KX279683 & 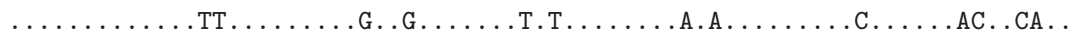 \\
\hline
\end{tabular}

FIGURE 2: Alignment of the sequences obtained with the 16S rRNA FRET-qPCR we used in our study and those of Anaplasma spp. in GenBank. "." denotes the identical nucleotide sequence to that of A. bovis. Organisms with GenBank accession numbers identified in the study are in red.

northern provinces (Heilongjiang, Beijing, Inner Mongolia, and Xinjiang).

Although we obtained relatively large numbers of animals positive by FRET-qPCR for the 16S rRNA of Anaplasma spp., we were only able to amplify a small number of these with the gltA primers. We presume this was because of low parasitemia in affected animals and different numbers of target sequences for the PCRs [25], since we could only amplify the gltA from animals with high copy numbers in the 16S rRNA FRETqPCR. It might also, however, have been because of different sensitivities of the PCRs we used as has been described before with molecular detection of different genes in Anaplasma spp. $[10,25,26]$.

The one Anaplasma we definitively identified with the gltA PCR was A. marginale which is the agent of bovine anaplasmosis, a very common disease of cattle around the world in tropical and subtropical countries [27]. Infections are mainly transmitted by Rhipicephalus microplus and although most infections are subclinical, there can be fever and severe anemia resulting in production losses from decreased milk production and abortion. Studies in China have shown that the organism can be found in Rhipicephalus 


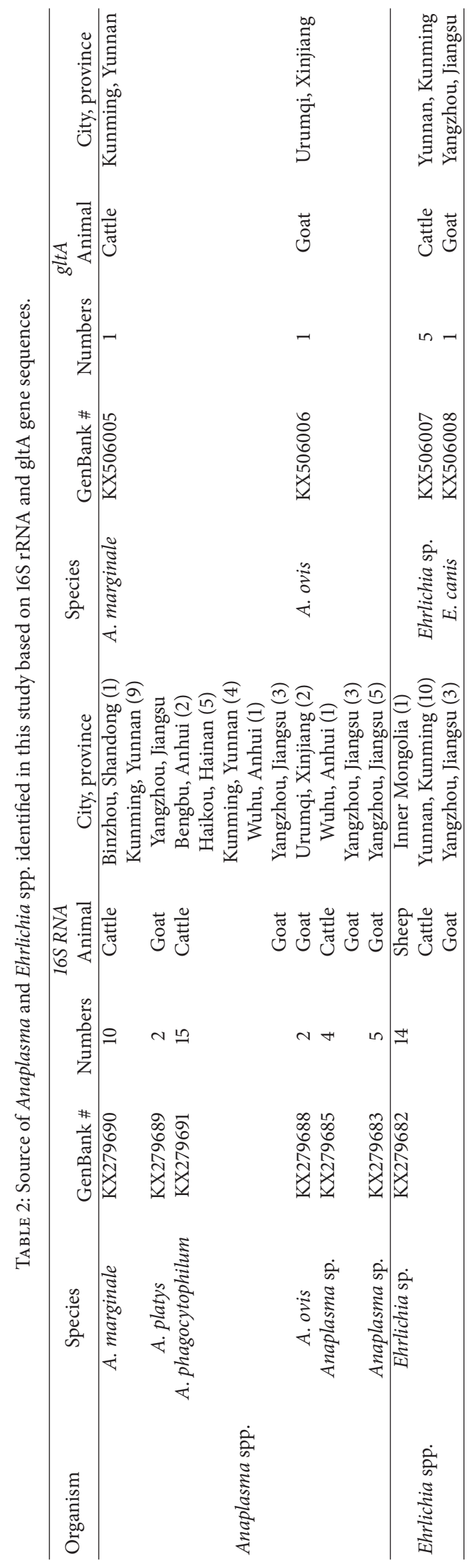




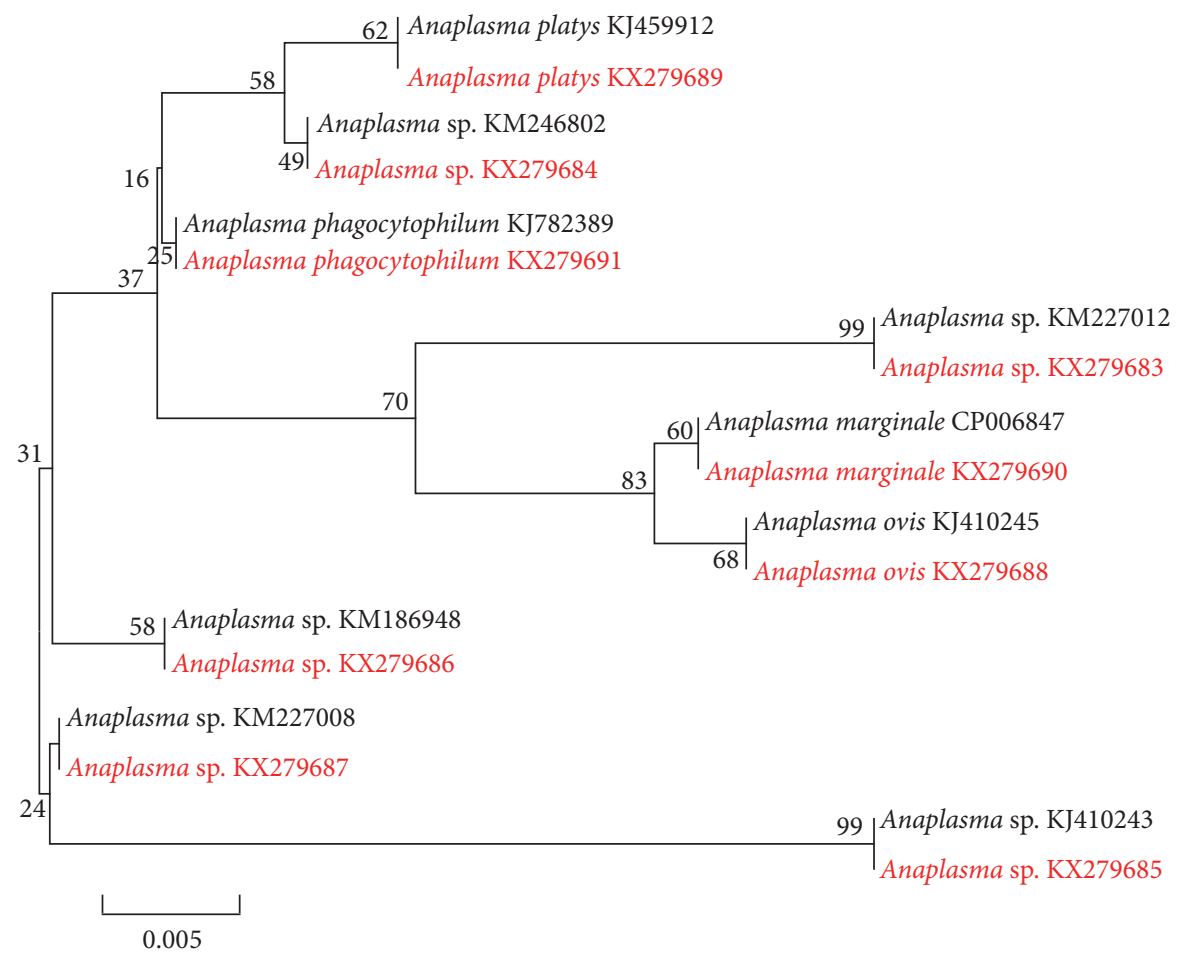

FIgURE 3: Phylogenetic comparison of Anaplasma spp. from ruminants in this study. The 16S rRNA sequences (in red font and accession number) are compared with those of other representing Anaplasma spp. (in black font and accession number). Branch lengths are measured in nucleotide substitutions and numbers show branching percentages in bootstrap replicate. Scale bar represents the percent sequence diversity.

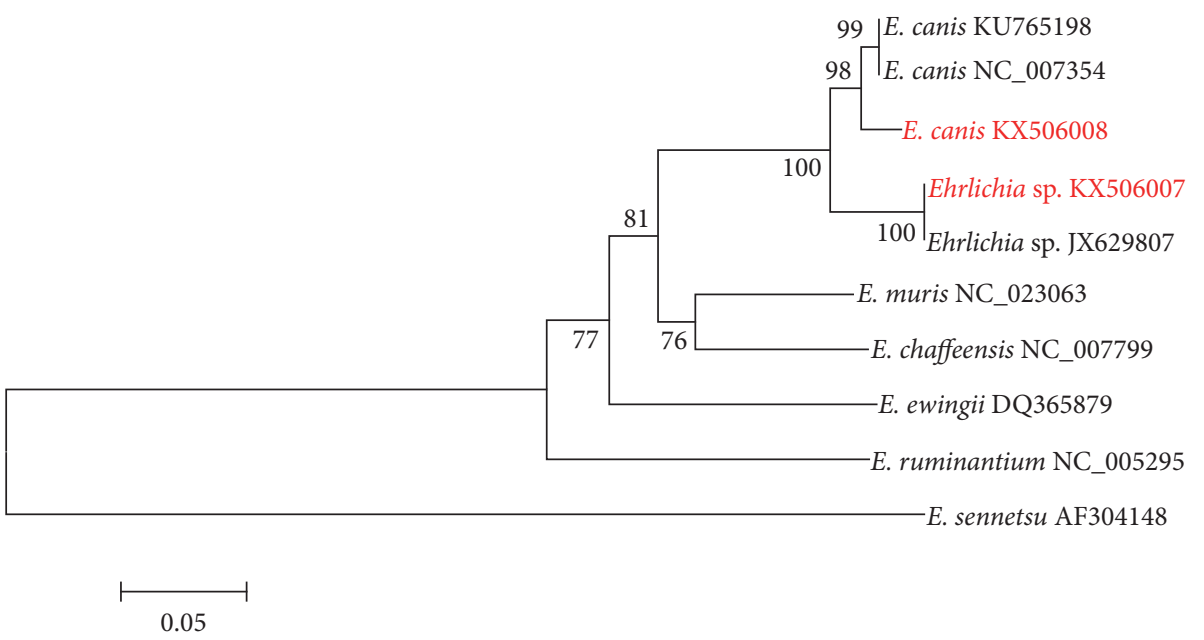

Figure 4: Phylogenetic comparison of Ehrlichia spp. from ruminants in this study. The gltA sequences (in red font and accession number) are compared with those of other representing Ehrlichia spp. (in black font and accession number). Branch lengths are measured in nucleotide substitutions and numbers show branching percentages in bootstrap replicate. Scale bar represents the percent sequence diversity.

(Boophilus) microplus [28] and also that the organism might be transmitted by Hyalomma asiaticum [5]. The organism appears to be widespread in domestic ruminants in China and it has been reported to be a relatively common infection of cattle in southern and northern China $[5,15,29,30]$.

The other Anaplasma we definitively identified, A. ovis, has also been reported previously in China in goats (15\%) from central and southern China [1] and in sheep and goats $(41 \%)$ in Henan and Xinjiang [6, 7]. This organism is the agent of ovine anaplasmosis which can be transmitted by Dermacentor nuttalli, Hyalomma asiaticum kozlovi, and Rhipicephalus pumilio in China where infections mostly result in subclinical anemia in indigenous animals [31]. Recently, the organism has been shown to infect humans [32] as has a closely related organism in China, putatively named "Anaplasma capra" [33]. 
Although we were not able to use glt $A$ sequencing to definitively identify most of the Anaplasma spp. we detected with our $16 S$ rRNA FRET-qPCR, we could confirm the accuracy of the qPCR in detecting $A$. marginale and $A$. ovis. Anaplasma marginale was one of the most common Anaplasma species we detected with the 16S rRNA FRETqPCR along with $A$. phagocytophilum which has also already been described in China where it appears to be common in ruminants $[7,11,12,34]$. Anaplasma phagocytophilum is the agent of tick-borne fever of ruminants and is transmitted by Dermacentor silvarum, Haemaphysalis concinna, H. longicornis, and Ixodes persulcatus in China $[35,36]$. The organism is now known to infect a wide variety of domestic and wild animals and is the agent of human granulocytic anaplasmosis [27].

Other Anaplasma spp. we appear to have identified based on their $16 \mathrm{~S}$ rRNA sequences include A. platys, the agent of infectious canine cyclic thrombocytopenia [3], which has been described in dogs in Asia $[24,37]$ and in sika deer, goats, and cattle in China [4, 19]. The remaining two Anaplasma spp. we appear to have found are as yet only poorly characterized with Anaplasma sp. (KM227012) first reported in Procapra gutturosa, the Mongolian gazelle, in China and appear to be most closely related to A. ovis and A. centrale [38]. Anaplasma sp. BL126-13 (KJ410243) has only been identified in a Hyalomma asiaticum from Xinjiang [39]. As we found that these poorly characterized organisms seem to occur relatively commonly, especially in goats, it would seem appropriate that they should be studied further as they could be important pathogens.

A number of Ehrlichia spp. have been described in China, E. chaffeensis, E. canis, E. platys, E. ewingii (granulocytic ehrlichial agent), and also a novel species closely related to E. chaffeensis and A. marginale $[14,15,33,40]$. The most important Ehrlichia species infecting ruminants, E. ruminantium, is restricted to Africa and some Caribbean islands [19] and has not been reported in China. Of the other Ehrlichia known to infect ruminants (summarized in Zhang et al., 2015 [19]), we only found evidence of infection with E. canis in the domestic ruminants we studied in China. Although E. canis is best known as a very common dog pathogen around the world, infections have also been described in people [41] and in cats [42], and there is thus growing evidence that E. canis has a wider host range than previously thought $[2,19,43]$. Our finding of E. canis and closely related organisms in a goat and cattle in China further supports this evidence and is consistent with the findings of a study showing that $E$. canis or very closely related organisms are present in domestic ruminants in the Caribbean [19] and also a study showing that E. canis occurs in sika deer in China [4]. Further studies are underway in our laboratory to determine the pathogenicity of $E$. canis in domestic ruminants.

In summary, we found DNA of Anaplasma spp. and Ehrlichia spp. relatively common in the blood of the goats ( $81.1 \%$ and $1.1 \%$, resp.), cattle (13.7\% and $3.6 \%$, resp.), sheep (11.7\% and $1.8 \%$, resp.), and water buffaloes $(6.9 \%$ and $0 \%$, resp.) we studied from China. Further, our data from 12 provinces show that a wide range of Anaplasma spp. and Ehrlichia spp. occur in ruminants in China and further larger scale studies are indicated to determine more accurate prevalence data for these agents and their impact on health and production. The low copy numbers we commonly found indicate that chronic infections are common and this did not enable us to obtain reliable multigene sequence data from most samples. It would appear best, then, for future studies on the presence of Anaplasma and Ehrlichia spp. to rather be conducted on organisms cultured from infected animals. Ticks should also be considered for such studies as they generally contain relatively high numbers of Anaplasma and Ehrlichia spp. (2,530 to 970,000/positive tick) [25].

\section{Competing Interests}

The authors declare that they have no competing interests.

\section{Authors' Contributions}

Chengming Wang, Haixiang Qiu, Jilei Zhang, and Patrick John Kelly designed the study. Haixiang Qiu, Jilei Zhang, Qinghua Luo, Yi Yang, Yongjiang Mao, Zhangping Yang, Jing $\mathrm{Li}$, and Hongzhuan $\mathrm{Wu}$ performed the experiment. Chengming Wang, Haixiang Qiu, Jilei Zhang, and Patrick John Kelly wrote the manuscript. All authors read and approved the final manuscript.

\section{Acknowledgments}

This project was supported by the National Natural Science Foundation of China (Grants 31272575 and 32472225) and by the Priority Academic Program Development of Jiangsu Higher Education Institutions.

\section{References}

[1] Z. Liu, M. Ma, Z. Wang et al., "Molecular survey and genetic identification of Anaplasma species in goats from central and southern China," Applied and Environmental Microbiology, vol. 78, no. 2, pp. 464-470, 2012.

[2] X.-J. Yu, J. W. McBride, and D. H. Walker, "Restriction and expansion of Ehrlichia strain diversity," Veterinary Parasitology, vol. 143, no. 3-4, pp. 337-346, 2007.

[3] P. J. Kelly, H. Lucas, C. Yowell et al., "Ehrlichia ruminantium in Amblyomma variegatumand domestic ruminants in the Caribbean," Journal of Medical Entomology, vol. 48, no. 2, pp. 485-488, 2011.

[4] Y. Li, Z. Chen, Z. Liu et al., "Molecular survey of Anaplasma and Ehrlichia of red deer and sika deer in Gansu, China in 2013," Transboundary and Emerging Diseases, vol. 63, no. 6, pp. e228e236, 2016.

[5] L. Zhang, Y. Wang, D. Cai et al., "Detection of Anaplasma marginale in Hyalomma asiaticum ticks by PCR assay," Parasitology Research, vol. 112, no. 7, pp. 2697-2702, 2013.

[6] Y. Zhang, Y. Lv, Y. Cui et al., "First molecular evidence for the presence of Anaplasma DNA in milk from sheep and goats in China," Parasitology Research, vol. 115, no. 7, pp. 2789-2795, 2016.

[7] J. Yang, Y. Li, Z. Liu et al., "Molecular detection and characterization of Anaplasma spp. in sheep and cattle from Xinjiang, northwest China," Parasites and Vectors, vol. 8, article 108, 2015. 
[8] L. Zhan, W.-C. Cao, J.-F. Jiang et al., "Anaplasma phagocytophilum in livestock and small rodents," Veterinary Microbiology, vol. 144, no. 3-4, pp. 405-408, 2010.

[9] L. Zhang, H. Liu, B. Xu et al., "Anaplasma phagocytophilum infection in domestic animals in ten provinces/cities of China," The American Journal of Tropical Medicine and Hygiene, vol. 87, no. 1, pp. 185-189, 2012.

[10] J. Yang, Z. Liu, Q. Niu et al., "Evaluation of different nested PCRs for detection of Anaplasma phagocytophilum in ruminants and ticks," BMC Veterinary Research, vol. 12, article 35, 2016.

[11] L. Zhang, F. Cui, L. Wang et al., "Investigation of anaplasmosis in Yiyuan County, Shandong Province, China," Asian Pacific Journal of Tropical Medicine, vol. 4, no. 7, pp. 568-572, 2011.

[12] Z. Yaxue, J. Hongtao, W. Qiuyue et al., "Molecular detection of anaplasma phagocytophilum in ixodid ticks in Hebei Province, China," Vector-Borne and Zoonotic Diseases, vol. 11, no. 10, pp. 1323-1327, 2011.

[13] J. Yang, Z. Liu, G. Guan et al., "Prevalence of Anaplasma phagocytophilum in ruminants, rodents and ticks in Gansu, north-western China," Journal of Medical Microbiology, vol. 62, no. 2, pp. 254-258, 2013.

[14] X.-C. Zhang, L.-X. Zhang, W.-H. Li et al., "Ehrlichiosis and zoonotic anaplasmosis in Suburban Areas of Beijing, China," Vector-Borne and Zoonotic Diseases, vol. 12, no. 11, pp. 932-937, 2012.

[15] B. Wen, R. Jian, Y. Zhang, and R. Chen, "Simultaneous detection of Anaplasma marginale and a new Ehrlichia species closely related to Ehrlichia chaffeensis by sequence analyses of $16 \mathrm{~s}$ ribosomal DNA in Boophilus microplus ticks from Tibet," Journal of Clinical Microbiology, vol. 40, no. 9, pp. 3286-3290, 2002.

[16] Y. Yang, Y. Mao, P. Kelly et al., "A pan-Theileria FRET-qPCR survey for Theileria spp. in ruminants from nine provinces of China," Parasites and Vectors, vol. 7, no. 1, article 413, 2014.

[17] Y. Yang, W. Fan, Y. Mao et al., "Bovine leukemia virus infection in cattle of China: association with reduced milk production and increased somatic cell score," Journal of Dairy Science, vol. 99, no. 5, pp. 3688-3697, 2016.

[18] P. J. Kelly, C. Xu, H. Lucas et al., "Ehrlichiosis, babesiosis, anaplasmosis and hepatozoonosis in dogs from St. Kitts, West Indies," PLoS ONE, vol. 8, no. 1, Article ID e53450, 2013.

[19] J. Zhang, P. Kelly, W. Guo et al., "Development of a generic Ehrlichia FRET-qPCR and investigation of ehrlichioses in domestic ruminants on five Caribbean islands," Parasites and Vectors, vol. 8, article 506, 2015.

[20] L. Wei, P. Kelly, J. Zhang et al., "Use of a universal hydroxymethylbilane synthase (HMBS)-based PCR as an endogenous internal control and to enable typing of mammalian DNAs," Applied Microbiology and Biotechnology, vol. 98, no. 12, pp. 5579-5587, 2014.

[21] A. D. Loftis, W. K. Reeves, J. P. Spurlock et al., "Infection of a goat with a tick-transmitted Ehrlichia from Georgia, U.S.A., that is closely related to Ehrlichia ruminantium," Journal of Vector Ecology, vol. 31, no. 2, pp. 213-223, 2006.

[22] A. C. Cruz, E. Zweygarth, M. F. B. Ribeiro et al., "New species of Ehrlichia isolated from Rhipicephalus (Boophilus) microplus shows an ortholog of the E. canis major immunogenic glycoprotein $g p 36$ with a new sequence of tandem repeats," Parasites and Vectors, vol. 5, article 291, 2012.

[23] H. Inokuma, P. Brouqui, M. Drancourt, and D. Raoult, "Citrate synthase gene sequence: a new tool for phylogenetic analysis and identification of Ehrlichia," Journal of Clinical Microbiology, vol. 39, no. 9, pp. 3031-3039, 2001.

[24] A. Kongklieng, T. Thanchomnang, P. M. Intapan et al., "Detection of Ehrlichia canis in canine blood samples by realtime fluorescence resonance energy transfer (FRET) PCR and melting curve analysis," The Southeast Asian journal of tropical medicine and public health, vol. 45, no. 5, pp. 1149-1156, 2014.

[25] C. B. da Silva, M. S. Pires, J. A. Vilela et al., "A new quantitative PCR method for the detection of Anaplasma platys in dogs based on the citrate synthase gene," Journal of Veterinary Diagnostic Investigation, vol. 28, no. 5, pp. 529-535, 2016.

[26] J. Stańczak, S. Cieniuch, A. Lass, B. Biernat, and M. Racewicz, "Detection and quantification of Anaplasma phagocytophilum and Babesia spp. in Ixodes ricinus ticks from urban and rural environment, northern Poland, by real-time polymerase chain reaction," Experimental and Applied Acarology, vol. 66, no. 1, pp. 63-81, 2015.

[27] P. Aubry and D. W. Geale, "A review of Bovine anaplasmosis," Transboundary and Emerging Diseases, vol. 58, no. 1, pp. 1-30, 2011.

[28] B. Wen, W. Cao, and H. Pan, "Ehrlichiae and ehrlichial diseases in China," Annals of the New York Academy of Sciences, vol. 990, pp. 45-53, 2003.

[29] Z. Zhou, K. Nie, C. Tang et al., "Phylogenetic analysis of the genus Anaplasmain Southwestern China based on 16S rRNA sequence," Research in Veterinary Science, vol. 89, no. 2, pp. 262$265,2010$.

[30] Z. Liu, J. Luo, Q. Bai, M. Ma, G. Guan, and H. Yin, "Amplification of 16S rRNA genes of Anaplasma species in China for phylogenetic analysis," Veterinary Microbiology, vol. 107, no. 1-2, pp. 145-148, 2005.

[31] W. Lu, W. Lu, Q. Zhang, F. Yu, H. Dou, and H. Yin, "Ovine anaplasmosis in northwest China," Tropical Animal Health and Production, vol. 29, supplement 4, pp. 16S-18S, 1997.

[32] D. Chochlakis, I. Ioannou, Y. Tselentis, and A. Psaroulaki, "Human anaplasmosis and Anaplasma ovis variant," Emerging Infectious Diseases, vol. 16, no. 6, pp. 1031-1032, 2010.

[33] H. Li, Y.-C. Zheng, L. Ma et al., "Human infection with a novel tick-borne Anaplasma species in China: A Surveillance Study," The Lancet Infectious Diseases, vol. 15, no. 6, pp. 663-670, 2015.

[34] Z. Chen, Q. Liu, J.-Q. Liu et al., “Tick-borne pathogens and associated co-infections in ticks collected from domestic animals in central China," Parasites and Vectors, vol. 7, article 237, 2014.

[35] X. Y. Liu, Z. Q. Wu, Z. Z. Liu, R. X. Tang, C. Yan, and K. Y. Zheng, "Investigation and phylogenic analysis of anaplasma derived from hard ticks in Yiyuan County, Shandong Province," Progress in Veterinary Medicine, vol. 36, pp. 103-107, 2015.

[36] J.-F. Jiang, B.-G. Jiang, J.-H. Yu et al., “Anaplasma phagocytophilum infection in ticks, China-Russia border," Emerging Infectious Diseases, vol. 17, no. 5, pp. 932-934, 2011.

[37] A. Unver, Y. Rikihisa, M. Kawahara, and S. Yamamoto, "Analysis of $16 S$ rRNA gene sequences of Ehrlichia canis, Anaplasma platys, and Wolbachia species from canine blood in Japan," Annals of the New York Academy of Sciences, vol. 990, pp. 692698, 2003.

[38] Y. Li, Z. Chen, Z. Liu et al., "First report of Theileria and Anaplasma in the Mongolian gazelle, Procapra gutturosa," Parasites and Vectors, vol. 7, article 614, 2014.

[39] Y.-J. Kang, X.-N. Diao, G.-Y. Zhao et al., "Extensive diversity of Rickettsiales bacteria in two species of ticks from China and the evolution of the Rickettsiales," BMC Evolutionary Biology, vol. 14, no. 1, article 167, 2014. 
[40] P. Hua, M. Yuhai, T. Shide, S. Yang, W. Bohai, and C. Xiangrui, "Canine ehrlichiosis caused simultaneously by Ehrlichia canis and Ehrlichia platys," Microbiology and Immunology, vol. 44, no. 9, pp. 737-739, 2000.

[41] M. Perez, M. Bodor, C. Zhang, Q. Xiong, and Y. Rikihisa, "Human infection with Ehrlichia canis accompanied by clinical signs in Venezuela," Annals of the New York Academy of Sciences, vol. 1078, pp. 110-117, 2006.

[42] Í. A. Braga, L. G. F. dos Santos, A. L. T. Melo et al., "Hematological values associated to the serological and molecular diagnostic in cats suspected of Ehrlichia canis infection," Revista Brasileira de Parasitologia Veterinária, vol. 22, no. 4, pp. 470-474, 2013.

[43] D. M. Aguiar, T. F. Ziliani, X. Zhang et al., "A novel Ehrlichia genotype strain distinguished by the TRP36 gene naturally infects cattle in Brazil and causes clinical manifestations associated with ehrlichiosis," Ticks and Tick-Borne Diseases, vol. 5, no. 5, pp. 537-544, 2014. 


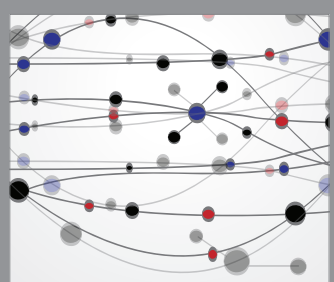

The Scientific World Journal
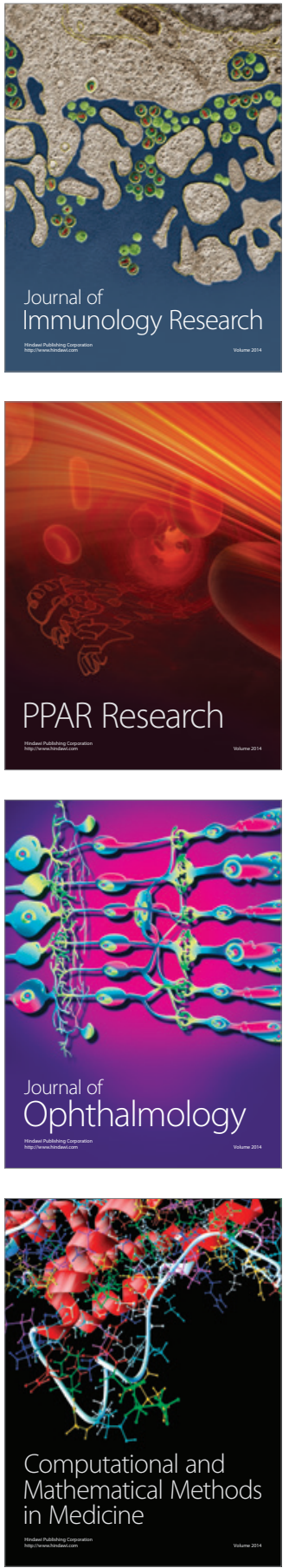

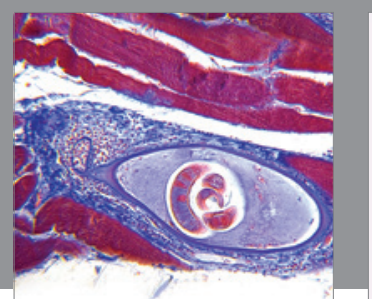

Gastroenterology Research and Practice

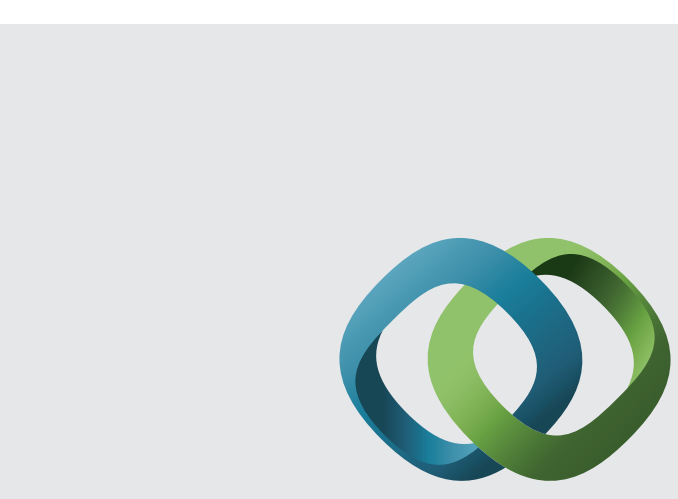

\section{Hindawi}

Submit your manuscripts at

http://www.hindawi.com
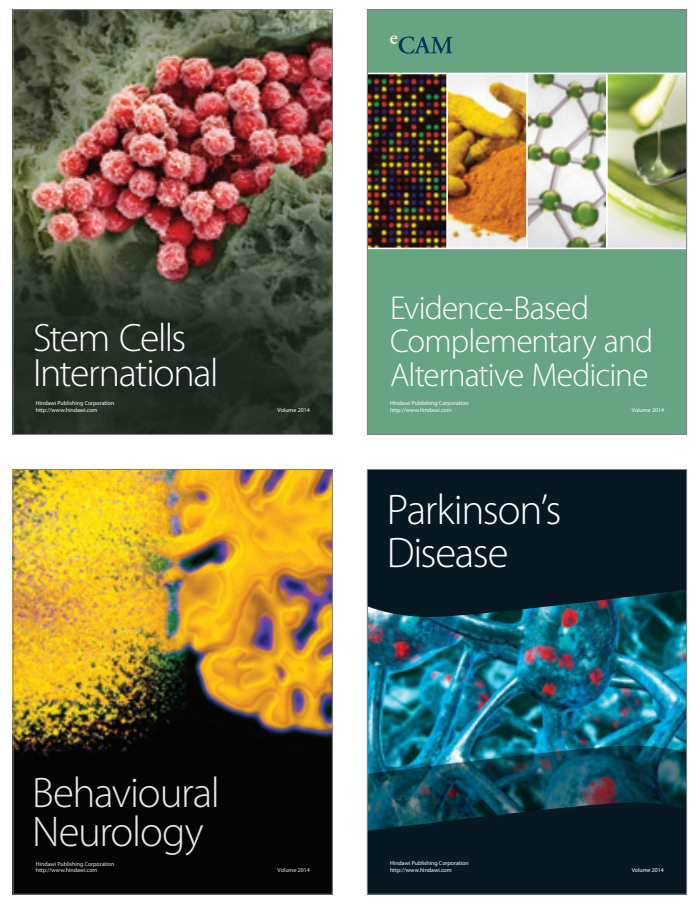
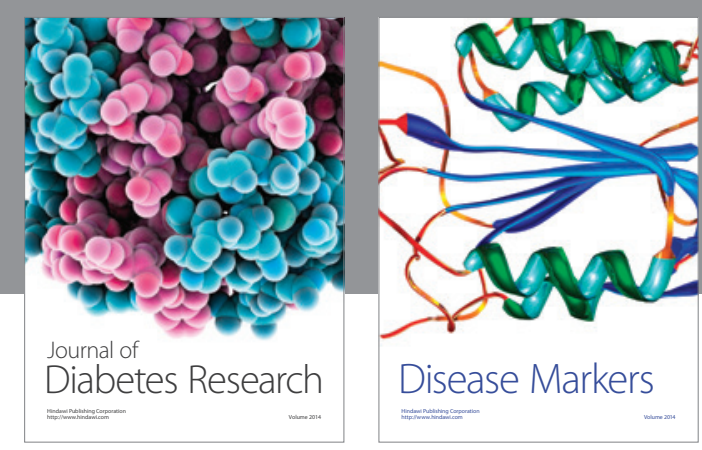

Disease Markers
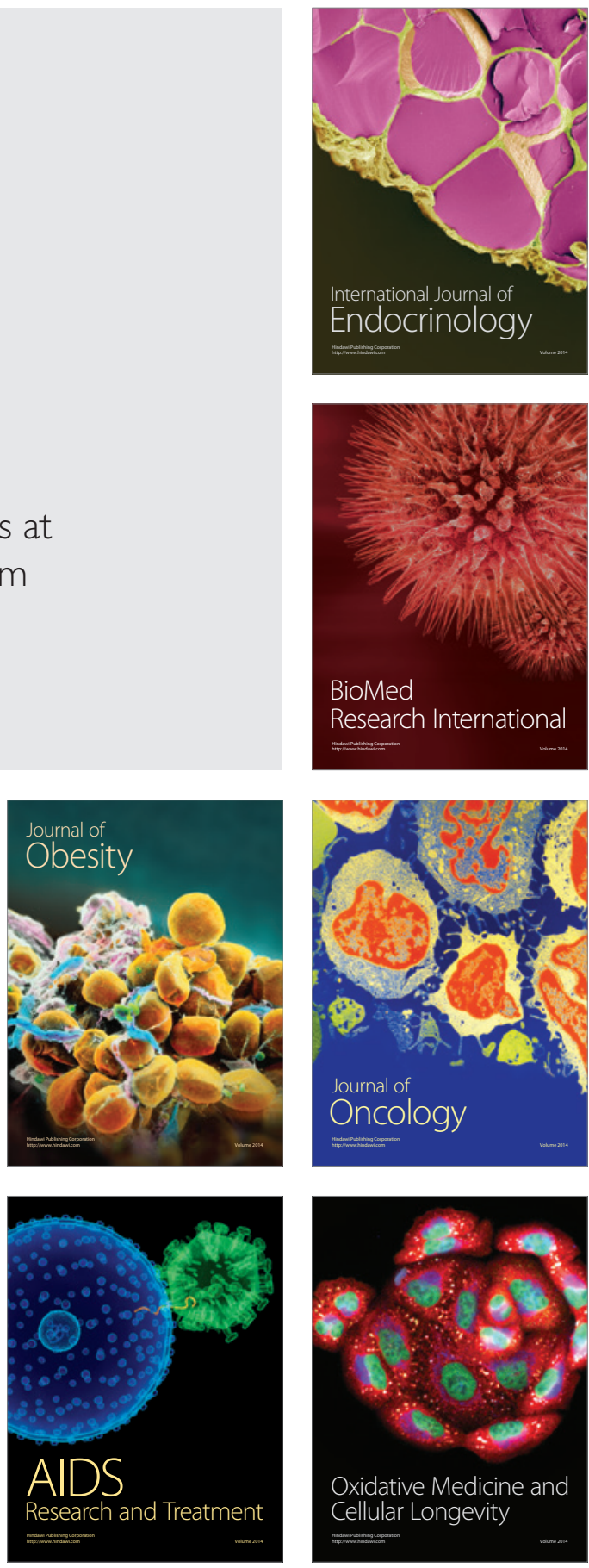